\title{
Measurement of Multiple Lumens in Glass Rods
}

\author{
W.J. Mershon* and J.R. Edwards** \\ *Camscan USA, Cranberry Township, PA, 16066 \\ **Becton Dickinson Accu-Glass, Saint Louis, MO, 63132
}

Glass rods with precision bores are to be used as metering devices in liquidflow delivery apparatus. Small voids in the glass melt can be unintentionally drawn into additional lumens through a segment of glass to be used as a flow restrictor, changing the rate at which fluid is delivered by the device. The current in-process inspection method uses an optical microscope and stage micrometer to measure the diameter of the large central bore, neglecting other lumens.

The customer decided to seek a SEM/Image analysis solution based on the need to more easily resolve lumens from glass and to measure lumens down to 2 microns in diameter. The customer needed a qualified measuring gage, so they insisted on evaluating performance based on a study of gage capability known as gage repeatability and reproducibility. Reproducibility and repatability are elements of gage variation [1]. A gage has good reproducibility if the measurement does not depend on the person who uses it. A gage has good repeatability if it returns the same value each time a specimen is measured. A gage study, or reproducibility and repeatability (R\&R) study, measures gage variation. The study involved measuring the same dimension of three identical parts, ten times each by three different operators. An analysis of the distribution of measurements determines the variance due to Appraiser Variation (AV), and Equipment Variation (EV). In this case, the dimension measured was the total area of lumens in the rod, net of the central bore. An acceptable R\&R is generally considered to be less than $10 \%$ when the AV and EV ae expressed as a percentage of the tolerance for the dimension being measured.

The system consists of a Tescan Vega SEM model TS5135MM, which includes low vacuum operation, a motorized computer-controlled stage and integrated image analysis software. The working conditions are $20 \mathrm{kV}$ accelerating voltage, 16 Pascals chamber pressure, $14 \mathrm{~mm}$ working distance, 600pA probe current, and a magnification of 500X. The glass rods were scored, snapped and then immediately placed into the SEM chamber to minimize contamination.

The sample was defined in the image analysis software as a circle with a diameter slightly larger than the outside diameter (OD) of the rod so that lumens all the way out to the OD could be detected and measured. This typically resulted in a matrix of 9X9 fields-of-view. The signal levels were adjusted so that the glass at the center of the rod was just less than maximum gray level and so that large lumens near the center of the rod were at minimum gray level. Images were acquired and processed simultaneously to speed up the analysis and each image was smoothed with a median filter before thresholding and object detection. The resulting objects were then classified by filtering them based on a combination of size, aspect ratio, shape factor and average brightness to eliminate artifacts such as dirt, particles of glass, edge effects at the OD and segments of fractures that were created during preparation of the rods. Any object could be revisited by double clicking on the displayed data for the object, which instructed the microscope to re-position the object in the fieldof-view. 
The Vega SEM/Image analysis system achieved less than $10 \%$ gage $\mathrm{R} \& \mathrm{R}$, which allows the user to accept the results in their SPC. The image analysis was able to identify all 2 micron diameter lumens even if they were at the OD, while at the same time rejecting all of the common artifacts. The system achieved a cycle time of less than 10 minutes, which included evacuating the microscope, image alignment, image analysis setup and execution, object review, and data archiving. This level of productivity allows the Vega SEM to be placed in the production environment where rods can be sampled continuously and immediate feedback can be given on the production process.

\section{References}

[1] "Measurement Systems Analysis, Reference Manual", 2nd Edition, copyright 1990, Chrysler Corporation, Ford Motor Company, General Motors Corporation.
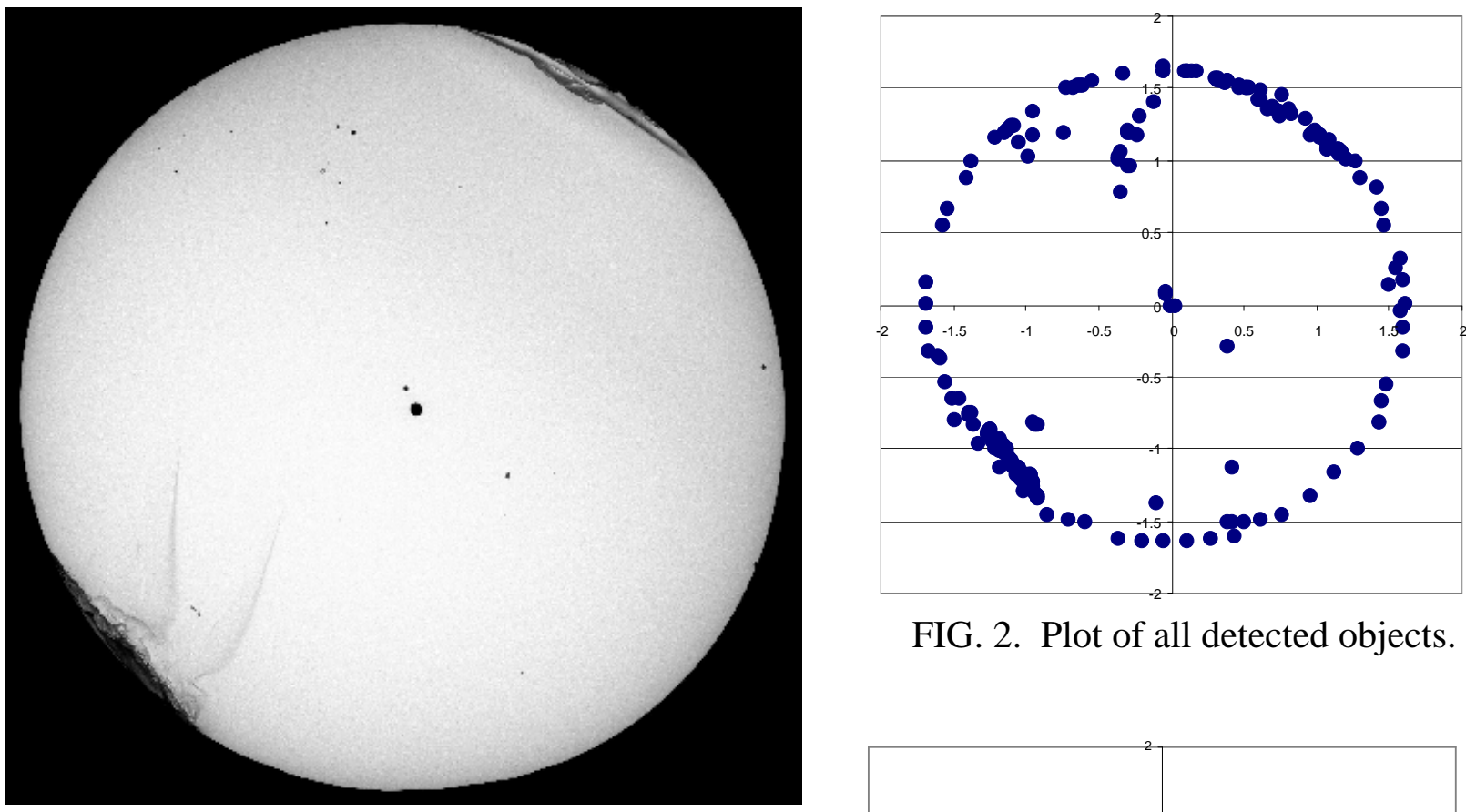

FIG. 2. Plot of all detected objects.

FIG. 1. A typical rod with a central bore, unintentional lumens, debris, and topography created by breaking the rod during preparation.

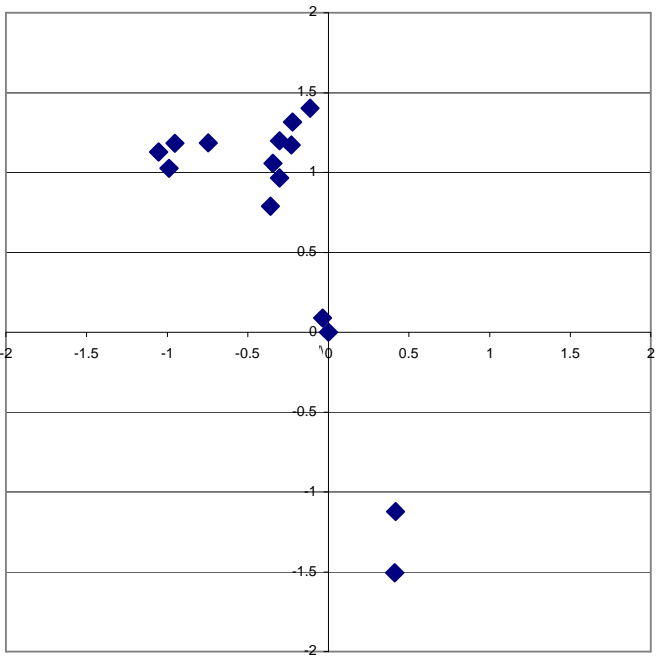

FIG. 3. Plot of filtered objects showing only unintentional lumens. 